\title{
XperCT Sharpening Reconstruction for Cone-Beam Computed Tomography Guided Lung and Bone Interventions
}

\author{
Christopher M. Murphy ${ }^{1} \quad$ L. Ray Ramoso ${ }^{1} \quad$ Eric J. Monroe ${ }^{1}$
}

1Division of Interventional Radiology, Department of Radiology,

Address for correspondence Eric J. Monroe, MD, Division of Seattle Children's Hospital, Seattle, Washington, United States

Interventional Radiology, Department of Radiology, Seattle Children's Hospital, 4800 Sand Point Way NE, M/S R-5417, Seattle, WA 98105, United States (e-mail: eric.monroe@seattlechildrens.org).

\begin{abstract}
Keywords

- $\mathrm{CBCT}$

- interventional radiology

- osteoid osteoma

- ablation

- biopsy

C-arm cone-beam computed tomography $(\mathrm{CBCT})$ is a valuable tool for three-dimensional navigation and mapping in the interventional radiology suite owing to its flexible gantry positioning, real-time three-dimensional volume acquisition, and reduced contrast and radiation use. Reports of CBCT-guided bone and lung interventions are relatively infrequent, however, possibly due in part to the lack of dedicated bone and lung reconstruction algorithms and concerns regarding insufficient lesion conspicuity. Two cases of an ad hoc intraprocedural CBCT sharpening reconstruction are presented in this article.
\end{abstract}

\section{Introduction}

C-arm cone beam computed tomography (CBCT) has been shown to have significant utility for procedural guidance., ${ }^{1,2}$ However, the adoption of $\mathrm{CBCT}$ as an adjunctive imaging modality in some arenas has been slowed by image quality limits and concerns over radiation dosing. ${ }^{3}$ Particularly in artifact-prone areas such as lung and bone, limits in image detail may encourage the operator to prefer standard computed tomography (CT), ultrasound, magnetic resonance imaging (MRI), or fluoroscopic guidance. Early experience, however, suggests that CBCT provides effective image guidance for bone ${ }^{4}$ and lung interventions. ${ }^{5-7}$

Recent advances in commercially available CBCT systems promise to improve image quality through reduction of noise, decreased radiation exposure, ${ }^{4}$ and artifact mitigation. The use of $\mathrm{CBCT}$ in guidance for pediatric procedures is likely to increase with reduced radiation dose and improved image detail. Accordingly, this technical report provides a demonstration of improved bone and lung lesion detection in pediatric patients using the Philips XperCT with XperGuide navigation (Philips) where dedicated bone and lung reconstruction algorithms are not yet commercially available. This novel combination of built-in reconstruction algorithms provides increased lesion conspicuity in a sample set of pediatric osteoid osteomas and lung nodules. Evaluation of these algorithms may provide opportunities for further research and use of $\mathrm{CBCT}$ in the interventional setting.

\section{Technique}

Written informed consent was obtained from the patients' parents after a discussion of risks, benefits, and alternatives of the procedure. Planning and procedural imaging were performed in a Philips FD20 angiography suite (aSi detector with CsI scintillator, $40 \times 30 \mathrm{~cm}$ detector) with Allura Clarity image optimization. A planning CBCT was performed through the body, with no lateral collimation and limited $\mathrm{z}$-axis coverage for the region known to contain the target lesion based on prior imaging. Using the XperCT workstation interface, "New Reconstruction" is selected. The reconstruction volume size is reduced to include the target lesion and percutaneous access route. Preset algorithms of "Stent" and "BMI Noise Reduction" are selected with a $512 \times 512$ matrix.
DOI https://doi.org/

$10.1055 / \mathrm{s}-0040-1705265$

ISSN 2457-0214.
Copyright $@ 2020$ by Indian Society of Vascular and Interventional Radiology
License terms

(ㅇ)( $\Theta \circledast$ 



Fig. 1 A 17-year-old male presented with right cuboid osteoid osteoma. Axial cone-beam computed tomography images (a) before and (b) after the sharpening reconstruction demonstrate the osteoid osteoma (white arrow) as well as the resultant increase in the surrounding trabecular detail.
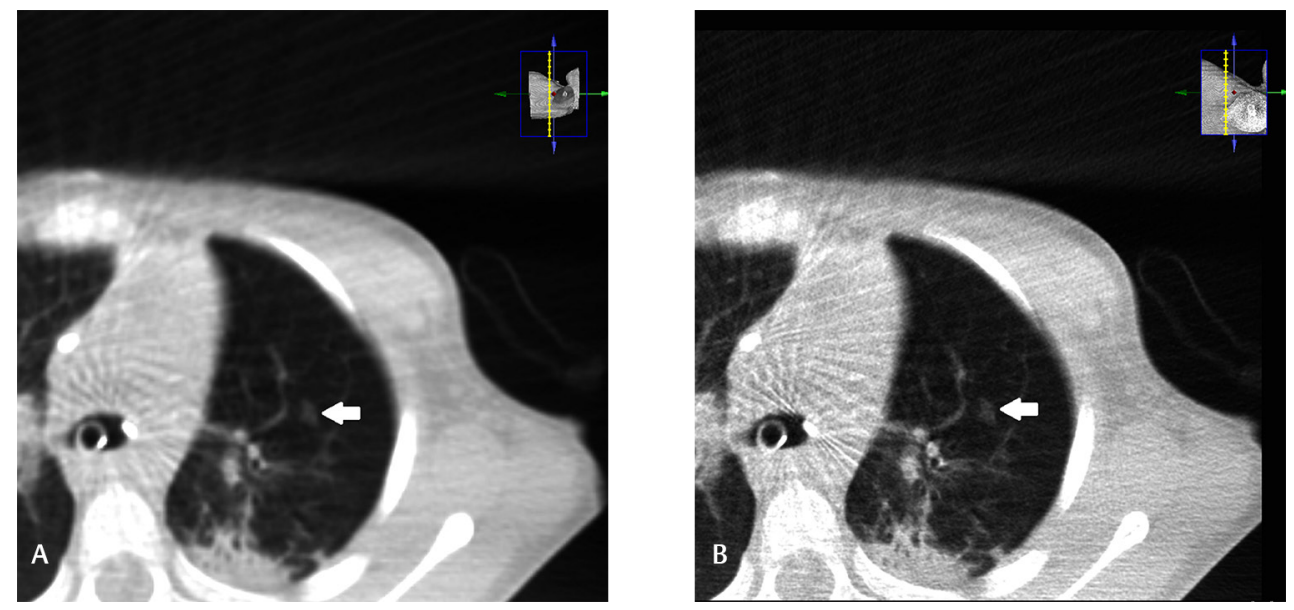

Fig. 2 A 5-year-old female presented with an indeterminate left upper lobe pulmonary nodule. Axial cone-beam computed tomography images (a) before and (b) after the sharpening reconstruction demonstrate the lesion (white arrow) as well as the resultant increase in the surrounding parenchymal detail.

The reconstructed image set is then used to plan and perform the procedure with navigational overlay.

\section{Case 1}

A 17-year-old male presenting with debilitating pain in the right foot and ankle, partially relieved by nonsteroidal anti-inflammatory medications, underwent successful CBCT-guided percutaneous needle access and microwave ablation (-Fig. 1). Fluoroscopy time and air kerma were 3.4 minutes and $13 \mathrm{mGy}$, respectively.

\section{Case 2}

A 5-year-old female with a history of a right renal Wilms tumor post chemotherapy, resection, and flank radiation presented with a persistent subcentimeter left upper lobe ground-glass opacity lung nodule. Image-guided wire localization was requested to facilitate wedge resection and determine the ongoing treatment regimen. CBCT-guided wire localization was performed successfully ( - Fig. 2), followed by wedge resection demonstrating focal necrosis with histiocytic inflammation consistent with prior tumor and no active malignancy. Fluoroscopy time and air kerma were 2.1 minutes and $22 \mathrm{mGy}$, respectively.

\section{Discussion}

The field of application of CBCT is expanding as additional improvements in noise and artifact reduction are producing higher quality images. Improved operator ergonomics, real-time volumetric reconstructions, and comodal overlays provide the interventionalist an increasingly accurate and immediate description of the procedural landscape. In pediatrics, dose-reducing techniques have been shown to preserve image quality while reducing total radiation exposure. ${ }^{2}$ In pediatric osteoid osteomas, CBCT has demonstrated similar technical and clinical success, reduced radiation dose, and increased total room utilization time compared with conventional CT guidance. ${ }^{4}$ Similarly, reports have demonstrated 
reduced lung radiation doses in simulated pediatric lung interventions, ${ }^{6}$ with successful use in adult lung biopsies for guidance. ${ }^{7}$ Published data on use of $\mathrm{CBCT}$ in the pediatric lung interventions are comparatively limited.

The primary limitation to the adoption of CBCT in diagnostic and procedural arenas is image quality. ${ }^{3}$ While flat-panel detectors offer comparable spatial resolution to standard CT, characteristic artifacts and noise can limit detail in reconstructions. ${ }^{1}$ Methods to produce detailed imaging while minimizing radiation or contrast exposure in both pediatric and adult interventional procedures would be highly beneficial. Currently, commercially available systems such as Philips XperCT provide several postacquisition reconstruction options to mitigate noise and artifact in specific imaging scenarios.

This technical report demonstrates improvement in lesion detail in example cases of pediatric lung and bone lesions through a combination of two previously developed $\mathrm{CBCT}$ reconstruction routines. Noted incidentally, the adaptive filtering and multipass reconstruction algorithms employed were initially developed for the reduction of artifacts in high-attenuation areas (obese patients and metallic implants). ${ }^{8}$ Their combined effect has been shown in our series to improve visibility of lesions as compared with standard $\mathrm{CBCT}$ reconstructions, without additional radiation or contrast exposure. Further exploration of the interaction between these filtering algorithms on pediatric lung and bone $\mathrm{CBCT}$ acquisitions, including interobserver variability assessment, is needed.

\section{Conclusion}

Until dedicated reconstruction algorithms become commercially available, the ad hoc image sharpening algorithm presented may increase lesion conspicuity and operator confidence during CBCT-guided bone and lung interventions.

\section{Conflicts of Interest}

None.

\section{Acknowledgments}

None.

\section{References}

1 Wallace MJ, Kuo MD, Glaiberman C, Binkert CA, Orth RC, Soulez G; Technology Assessment Committee of the Society of Interventional Radiology. Three-dimensional C-arm conebeam CT: applications in the interventional suite. J Vasc Interv Radiol 2009;20(7, Suppl):S523-S537

2 Acord M, Shellikeri S, Vatsky S, et al. Reduced-dose C-arm computed tomography applications at a pediatric institution. Pediatr Radiol 2017;47(13):1817-1824

$3 \mathrm{Hu} \mathrm{YH,} \mathrm{Fueglistaller} \mathrm{R,} \mathrm{Myronakis} \mathrm{M,} \mathrm{et} \mathrm{al.} \mathrm{Physics} \mathrm{consider-}$ ations in MV-CBCT multi-layer imager design. Phys Med Biol 2018;63(12):125016

4 Perry BC, Monroe EJ, McKay T, Kanal KM, Shivaram G. Pediatric percutaneous osteoid osteoma ablation: CONE-beam CT with fluoroscopic overlay versus conventional CT guidance. Cardiovasc Intervent Radiol 2017;40(10):1593-1599

5 Shivaram GM, Gill AE, Monroe EJ, Koo KS, Hawkins CM. Conebeam computed tomography guidance with navigational overlay for percutaneous lung nodule biopsy. Pediatr Radiol 2018;8:1-5

6 Ben-Shlomo A, Cohen D, Bruckheimer E, et al. Comparing effective doses during image-guided core needle biopsies with computed tomography versus $\mathrm{C}$-arm cone beam CT using adult and pediatric phantoms. Cardiovasc Intervent Radiol 2016;39(5):732-739

7 Hwang HS, Chung MJ, Lee JW, Shin SW, Lee KS. C-arm conebeam CT-guided percutaneous transthoracic lung biopsy: usefulness in evaluation of small pulmonary nodules. AJR Am J Roentgenol 2010;195(6):W400-7

8 Prell D, Kalender WA, Kyriakou Y. Development, implementation and evaluation of a dedicated metal artefact reduction method for interventional flat-detector CT. Br J Radiol 2010;83(996):1052-1062

\section{Funding}

None. 